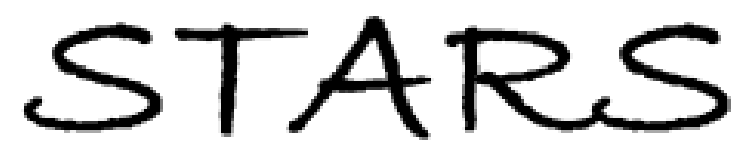

University of Central Florida

STARS

$1-1-2004$

\title{
Self-consistency of Vuks equations for liquid-crystal refractive indices
}

Jun Li

University of Central Florida

Shin-Tson Wu

University of Central Florida

Find similar works at: https://stars.library.ucf.edu/facultybib2000 University of Central Florida Libraries http://library.ucf.edu

This Article is brought to you for free and open access by the Faculty Bibliography at STARS. It has been accepted for inclusion in Faculty Bibliography 2000 s by an authorized administrator of STARS. For more information, please contact STARS@ucf.edu.

\section{Recommended Citation}

$\mathrm{Li}$, Jun and Wu, Shin-Tson, "Self-consistency of Vuks equations for liquid-crystal refractive indices" (2004). Faculty Bibliography 2000s. 4540.

https://stars.library.ucf.edu/facultybib2000/4540

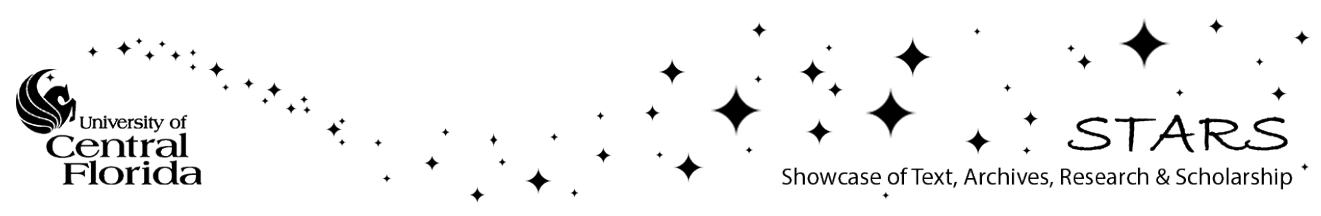




\section{Self-consistency of Vuks equations for liquid- crystal refractive indices}

Cite as: J. Appl. Phys. 96, 6253 (2004); https://doi.org/10.1063/1.1812356

Submitted: 27 May 2004 . Accepted: 08 September 2004 . Published Online: 22 November 2004

Jun $\mathrm{Li}$, and Shin-Tson Wu

\section{ARTICLES YOU MAY BE INTERESTED IN}

Extended Cauchy equations for the refractive indices of liquid crystals

Journal of Applied Physics 95, 896 (2004); https://doi.org/10.1063/1.1635971

Temperature effect on liquid crystal refractive indices

Journal of Applied Physics 96, 19 (2004); https://doi.org/10.1063/1.1757034

Infrared refractive indices of liquid crystals

Journal of Applied Physics 97, 073501 (2005); https://doi.org/10.1063/1.1877815

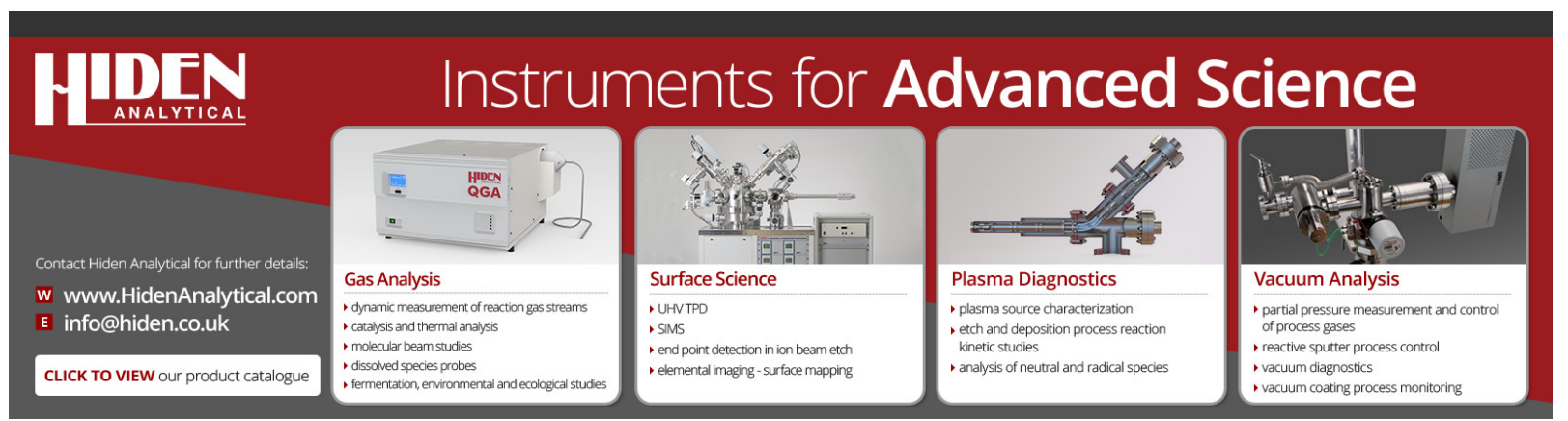




\title{
Self-consistency of Vuks equations for liquid-crystal refractive indices
}

\author{
Jun Li and Shin-Tson $\mathrm{Wu}^{\mathrm{a})}$ \\ College of Optics and Photonics, University of Central Florida, Orlando, Florida 32816
}

(Received 27 May 2004; accepted 8 September 2004)

\begin{abstract}
Vuks equations correlate the microscopic molecular polarizabilities to the macroscopic refractive indices of anisotropic crystalline materials. For anisotropic liquid crystals, the molecular polarizabilities are difficult to measure directly due to the short- and long-range interactions. We have converted Vuks equations into different forms. By measuring the refractive indices at different temperatures and wavelengths, the Vuks equations can be validated. Five liquid-crystal materials with refractive index ranging from $\sim 1.46$ to $\sim 1.86$ are used to validate the modified Vuks equations. The experimental results agree with the theory very well. Based on the Vuks equations, the molecular polarizabilities of 4-cyano-4-n-pentylbiphenyl are calculated. () 2004 American Institute of Physics. [DOI: 10.1063/1.1812356]
\end{abstract}

\section{INTRODUCTION}

Liquid crystals have been used extensively for displays, ${ }^{1}$ laser beam steering, ${ }^{2}$ and tunable photonic devices. ${ }^{3}$ Most liquid-crystal (LC) devices utilize electric field, optical field, or thermal-induced refractive index change to modulate light. ${ }^{4}$ The LC refractive indices are determined mainly by the molecular constituents, wavelength, and temperature. ${ }^{5}$ Since LC is an anisotropic molecular system involving the short- and long-range molecular interactions, its refractive index is quite different from that of an isotropic liquid.

About four decades ago, Vuks proposed a semiempirical model $^{6}$ which is analogous to the classical Clausius-Mossotti equation for correlating the microscopic molecular polarizabilities to the macroscopic refractive indices of some crystalline materials. Based on the Vuks model, several phenomenological models have been developed to describe the wavelength and temperature dependencies of the LC refractive indices. $^{7-14}$ In the Vuks model, an isotropic local field is assumed. Vuks validated his model by using the experimental data of several anisotropic crystals such as naphthalene, diphenyl, anthracene, and phenanthrene. ${ }^{6}$ Since the Vuks model has been used widely to describe the LC refractive indices, it is necessary to validate this model directly by using the experimental data of liquid-crystal materials.

To validate the Vuks equations for the LC molecular system is a challenging task because the LC molecular polarizabilities involve short-range and long-range interactions. In this article, we derive modified the Vuks equations and find that their validity can be easily examined by measuring the temperature- and wavelength-dependent refractive indices of liquid crystals. In Sec. II, we briefly discuss the derivation procedures. In Sec. III, the experimental method for measuring the refractive indices of liquid-crystal materials is briefly described. In Sec. IV, we describe the experimental results for validating Vuks equations using liquid-crystal materials. An excellent agreement between theory and experi-

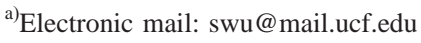

ment is obtained. Finally, we use Vuks equations to calculate the temperature-dependent polarizabilites of 4-cyano-4-npentylbiphenyl (5CB) at $\lambda=589 \mathrm{~nm}$.

\section{THEORY}

The classical Clausius-Mossotti equation correlates the permittivity $(\varepsilon)$ of an isotropic media with molecular polarizability $(\alpha)$ as follows: ${ }^{15}$

$$
\frac{\varepsilon-1}{\varepsilon+2}=\frac{4 \pi}{3} N \alpha .
$$

In Eq. (1), $N$ is the molecular packing density or number of molecules per unit volume. In the optical frequency regime, we substitute $\varepsilon=n^{2}$ and obtain the Lorentz-Lorenz equation: ${ }^{15}$

$$
\frac{n^{2}-1}{n^{2}+2}=\frac{4 \pi}{3} N \alpha .
$$

For an anisotropic LC, there are two principal refractive indices, $n_{e}$ and $n_{o}$, where $n_{e}$ and $n_{o}$ are refractive indices for the extraordinary ray and ordinary ray, respectively. In principle, each refractive index should be related to the corresponding molecular polarizabilities, $\alpha_{e}$ and $\alpha_{o}$. An early approach replaces both $n^{2}$ in Eq. (2) by $n_{e, o}^{2}$ and $\alpha$ by $\alpha_{e, o}{ }^{16}$ However, this model does not fit the experimental results well. Vuks made a bold assumption that the internal field in a crystal is the same in all directions, ${ }^{6}$

$$
E_{i}=\frac{\left\langle n^{2}\right\rangle+2}{3} E,
$$

where $E_{i}$ is the internal field, the average field that acts on a molecule, and $E$ is the macroscopic electric field. This assumption is later validated experimentally. ${ }^{7}$ With this assumption, Vuks derived the following equation for anisotropic media: ${ }^{6}$ 


$$
\frac{n_{e, o}^{2}-1}{\left\langle n^{2}\right\rangle+2}=\frac{4 \pi}{3} N \alpha_{e, o} .
$$

Equation (4) is different from Eq. (2) in two aspects: (1) the $n^{2}$ term in the denominator of Eq. (2) is replaced by $\left\langle n^{2}\right\rangle$ $=\left(n_{e}^{2}+2 n_{o}^{2}\right) / 3$, while the $n^{2}$ term in the numerator is replaced by $n_{e, o}^{2}$ and (2) the $\alpha$ is replaced by $\alpha_{e, o}$.

In Eq. (4), the macroscopic LC refractive indices $n_{e}$ and $n_{o}$ can be measured easily, however, the microscopic molecular polarizabilities $\alpha_{e}$ and $\alpha_{o}$ are difficult to determine directly. To validate Vuks equations, we rewrite Eq. (4) as follows:

$$
\begin{aligned}
& \frac{n_{e}^{2}-1}{\left\langle n^{2}\right\rangle+2}=\frac{4 \pi}{3} N \alpha_{e}, \\
& \frac{n_{o}^{2}-1}{\left\langle n^{2}\right\rangle+2}=\frac{4 \pi}{3} N \alpha_{o} .
\end{aligned}
$$

Multiplying both sides of Eq. (5b) by 2 and then add the corresponding terms with Eq. (5a), we obtain the following equation:

$$
\frac{\left(n_{e}^{2}+2 n_{o}^{2}\right)-3}{\left\langle n^{2}\right\rangle+2}=\frac{4 \pi}{3} N\left(\alpha_{e}+2 \alpha_{o}\right) .
$$

Equation (6) can be rewritten as

$$
\frac{\left\langle n^{2}\right\rangle-1}{\left\langle n^{2}\right\rangle+2}=\frac{4 \pi}{3} N\langle\alpha\rangle,
$$

where the average molecular polarizability is defined as $\langle\alpha\rangle=\left(\alpha_{e}+2 \alpha_{o}\right) / 3$. To evaluate Eq. (7) experimentally, we need to correlate $N\langle\alpha\rangle$ with another measurable macroscopic parameter, such as the average refractive index.

From Eq. (4), we can express $n_{e}$ and $n_{o}$ as follows: ${ }^{13,14}$

$$
\begin{aligned}
& n_{e}=\left[1+\frac{4 \pi N \alpha_{e}}{1-\frac{4}{3} \pi N\langle\alpha\rangle}\right]^{1 / 2}, \\
& n_{o}=\left[1+\frac{4 \pi N \alpha_{o}}{1-\frac{4}{3} \pi N\langle\alpha\rangle}\right]^{1 / 2} .
\end{aligned}
$$

When $N \alpha_{e, o}$ is small, the term $4 \pi N \alpha_{e, o} /(1-4 / 3 \pi N\langle\alpha\rangle)$ is small and Eq. (8) can be expanded into power series,

$$
n_{e, o} \approx 1+\frac{2 \pi N \alpha_{e, o}}{1-\frac{4}{3} \pi N\langle\alpha\rangle} .
$$

In the extreme case that $N \alpha_{i} \rightarrow 0$, the original Vuks equation [Eq. (4)] leads to $n_{e} \approx n_{o} \approx 1$. These results are consistent with Eq. (9). However, for most liquid crystals developed so far, their refractive indices are around 1.45-1.75. Therefore, the second term in the square root of Eq. (8) is larger than 1 but less than 2.1, the middle point is $\sim 1.5$. Under such a circumstance, Eq. (8) cannot be expanded into power series directly. In order to make a series expansion, we rewrite Eqs. (8a) and (8b) as

$$
n_{e}=\sqrt{2.5}\left[1+\frac{\left(x_{e}-1.5\right)}{2.5}\right]^{1 / 2},
$$

$$
\begin{aligned}
& n_{o}=\sqrt{2.5}\left[1+\frac{\left(x_{o}-1.5\right)}{2.5}\right]^{1 / 2}, \\
& x_{e, o}=\frac{4 \pi N \alpha_{e, o}}{1-\frac{4}{3} \pi N\langle\alpha\rangle} .
\end{aligned}
$$

The absolute value of $\left(x_{e, o}-1.5\right) / 2.5$ in Eqs. (10a) and (10b) is smaller than $\sim 0.2$. By expanding Eqs. (10a) and (10b) into power series and keeping the first two terms, we obtain

$$
\begin{aligned}
& n_{e} \approx \frac{7}{2 \sqrt{10}}+\frac{(2 \sqrt{10} / 5) \pi N \alpha_{e}}{1-\frac{4}{3} \pi N\langle\alpha\rangle}, \\
& n_{o} \approx \frac{7}{2 \sqrt{10}}+\frac{(2 \sqrt{10} / 5) \pi N \alpha_{o}}{1-\frac{4}{3} \pi N\langle\alpha\rangle} .
\end{aligned}
$$

During the series expansion, the second-order terms $(\sqrt{2.5} / 8)\left[\left(x_{e, o}-1.5 / 2.5\right)\right]^{2}$ in Eqs. (11a) and (11b) are neglected because they contribute less than $0.57 \%$ to the entire refractive index values. The expressions (11a) and (11b) presented here are more accurate than those we derived earlier. ${ }^{14}$

In Eq. (11), the two refractive indices are still coupled through $\langle\alpha\rangle$. Our goal is to correlate the microscopic quantity $N\langle\alpha\rangle$ with a macroscopic measurable quantity, such as $\langle n\rangle$. To do so, we express the molecular polarizabilities $\alpha_{e}$ and $\alpha_{o}$ as $^{13}$

$$
\begin{aligned}
& \alpha_{e}=\langle\alpha\rangle+\frac{2 S}{3}\left(\gamma_{e}-\gamma_{o}\right), \\
& \alpha_{o}=\langle\alpha\rangle-\frac{S}{3}\left(\gamma_{e}-\gamma_{o}\right) .
\end{aligned}
$$

Substituting Eqs. (12a) and (12b) back to Eqs. (11a) and (11b), respectively, we can express $n_{e}$ and $n_{o}$ in terms of $N$, $\langle\alpha\rangle$, and $\alpha_{e}-\alpha_{o}$ [which is equal to $S\left(\gamma_{e}-\gamma_{o}\right)$ ] by power expansions,

$$
\begin{aligned}
n_{e} \approx & \frac{7}{2 \sqrt{10}}+\frac{(2 \sqrt{10} / 5) \pi N\langle\alpha\rangle}{1-\frac{4}{3} \pi N\langle\alpha\rangle} \\
& +\frac{(4 \sqrt{10} / 15) \pi N S\left(\gamma_{e}-\gamma_{o}\right)}{1-\frac{4}{3} \pi N\langle\alpha\rangle}, \\
n_{e} \approx & \frac{7}{2 \sqrt{10}}+\frac{(2 \sqrt{10} / 5) \pi N\langle\alpha\rangle}{1-\frac{4}{3} \pi N\langle\alpha\rangle} \\
& -\frac{(2 \sqrt{10} / 15) \pi N S\left(\gamma_{e}-\gamma_{o}\right)}{1-\frac{4}{3} \pi N\langle\alpha\rangle} .
\end{aligned}
$$

Here, $\gamma_{e}-\gamma_{o}$ is the differential molecular polarizability in the crystalline state, ${ }^{17,18}$ and $S$ is the order parameter. In a temperature not too close to the clearing temperature $\left(T_{c}\right)$, the order parameter can be approximated as follows: ${ }^{19}$

$$
S=\left(1-T / T_{c}\right)^{\beta} .
$$

In Eq. (14), the exponent $\beta$ is a material constant. For many LC compounds, $\beta \sim 0.2$ is not too sensitive to the LC molecular structures. 
Based on Eqs. (13a) and (13b), we can calculate the average refractive index, which is defined as $\langle n\rangle=\left(n_{e}\right.$ $\left.+2 n_{o}\right) / 3$, and obtain the following equation:

$$
\langle n\rangle \approx \frac{7}{2 \sqrt{10}}+\frac{(2 \sqrt{10} / 5) \pi N\langle\alpha\rangle}{1-\frac{4}{3} \pi N\langle\alpha\rangle} .
$$

Equation (15) can be rearranged as follows:

$$
1-\frac{3}{\sqrt{10}\langle n\rangle-0.5} \approx \frac{4}{3} \pi N\langle\alpha\rangle .
$$

Equation (16) correlates $N\langle\alpha\rangle$ to the average refractive index $\langle n\rangle$. This is an important step as the microscopic quantity $N\langle\alpha\rangle$ can be determined experimentally by measuring the LC refractive indices. In order to compare with Eq. (16), we rewrite Eq. (7) as

$$
1-\frac{3}{\left\langle n^{2}\right\rangle+2}=\frac{4}{3} \pi N\langle\alpha\rangle .
$$

By comparing Eq. (16) with Eq. (17), we find

$$
\left\langle n^{2}\right\rangle+2 \approx \sqrt{10}\langle n\rangle-0.5 .
$$

The significance of Eq. (18) is that the sophisticated microscopic Vuks equation can now be validated by two simple macroscopic parameters $\left\langle n^{2}\right\rangle$ and $\langle n\rangle$. These two parameters can be obtained by measuring the individual refractive indices $\left(n_{e}\right.$ and $\left.n_{o}\right)$ of the liquid crystal. In Sec. IV, we will use the experimental data of five LC materials with birefringence ranging from 0.08 to 0.35 to validate Eq. (18).

\section{EXPERIMENT}

We measured the refractive indices of UCF-35 (a high birefringence LC mixture), 5CB, 4-cyano-4-n-pentylecyclohexane-phenyl (5PCH), MLC-6241-000 (a commercial low birefringence LC mixture), and UCF-280 $(\Delta \varepsilon<0)$ using a multiwavelength Abbe refractometer (Atago DR-M4) at $\lambda$ $=450,486,546,589,633$, and $656 \mathrm{~nm}$. The accuracy of the Abbe refractometer is up to the fourth decimal. For a given wavelength, we measured the refractive indices of these five LCs from 10 to $60{ }^{\circ} \mathrm{C}$. The temperature of the Abbe refractometer is controlled by a circulating constant-temperature bath (Atago Model 60-C3). The LC molecules are aligned perpendicular to the main prism surface of the Abbe refractometer by coating a surfactant comprising of $0.294 \mathrm{wt} \%$ hexadecyletri-methyle-ammonium bromide in methanol solution. Both $n_{e}$ and $n_{e}$ are obtained through a polarizing eyepiece. From our measurements, the clearing points of UCF35, 5CB, 5PCH, MLC-6241-000, and UCF-280 are 368.3, 306.6, 325.9, 373.7, and 339.2 K, respectively.

\section{RESULTS AND DISCUSSIONS}

We have measured the refractive indices of two LC compounds $(5 \mathrm{CB}$ and $5 \mathrm{PCH})$ and three eutectic mixtures [UCF35, MLC-6241-000, and UCF-280 $(\Delta \varepsilon<0)$ ] at various wavelengths and temperatures. Their raw data are listed in Tables I-V, respectively.

Figure 1 depicts the temperature-dependent birefrin-

\begin{tabular}{|c|c|c|c|c|c|c|}
\hline \multirow[b]{3}{*}{$T\left({ }^{\circ} \mathrm{C}\right)$} & \multicolumn{6}{|c|}{$\lambda(\mathrm{nm})$} \\
\hline & \multicolumn{2}{|c|}{546} & \multicolumn{2}{|c|}{589} & \multicolumn{2}{|c|}{633} \\
\hline & $n_{e}$ & $n_{o}$ & $n_{e}$ & $n_{o}$ & $n_{e}$ & $n_{o}$ \\
\hline 11.4 & 1.7566 & 1.5361 & 1.7448 & 1.5317 & 1.7361 & 1.5281 \\
\hline 12.3 & 1.7549 & 1.5362 & 1.7431 & 1.5316 & 1.7342 & 1.5274 \\
\hline 13.2 & 1.7531 & 1.5364 & 1.7414 & 1.5317 & 1.7328 & 1.5279 \\
\hline 14.2 & 1.7511 & 1.5364 & 1.7396 & 1.5317 & 1.7309 & 1.5279 \\
\hline 15.1 & 1.7493 & 1.5365 & 1.7378 & 1.5320 & 1.7289 & 1.5280 \\
\hline 16.1 & 1.7475 & 1.5370 & 1.736 & 1.5323 & 1.7271 & 1.5283 \\
\hline 17.0 & 1.7454 & 1.5373 & 1.734 & 1.5324 & 1.7253 & 1.5285 \\
\hline 18.4 & 1.7421 & 1.5374 & 1.7309 & 1.5327 & 1.7222 & 1.5288 \\
\hline 20.3 & 1.7378 & 1.5380 & 1.7264 & 1.5334 & 1.7181 & 1.5295 \\
\hline 22.5 & 1.7316 & 1.5394 & 1.7205 & 1.5343 & 1.7123 & 1.5305 \\
\hline 25.4 & 1.7232 & 1.5411 & 1.7124 & 1.5361 & 1.7038 & 1.5321 \\
\hline 27.2 & 1.7165 & 1.5429 & 1.7059 & 1.5380 & 1.6977 & 1.5336 \\
\hline 29.6 & 1.7056 & 1.5460 & 1.6954 & 1.5408 & 1.6876 & 1.5364 \\
\hline 32.6 & 1.6819 & 1.5544 & 1.6724 & 1.5487 & 1.6654 & 1.5443 \\
\hline 33.4 & 1.5940 & 1.5940 & 1.5872 & 1.5872 & 1.5820 & 1.5820 \\
\hline 34.7 & 1.5938 & 1.5938 & 1.5868 & 1.5868 & 1.5815 & 1.5815 \\
\hline 36.1 & 1.5927 & 1.5927 & 1.5859 & 1.5859 & 1.5809 & 1.5809 \\
\hline 38.0 & 1.5919 & 1.5919 & 1.5849 & 1.5849 & 1.5795 & 1.5795 \\
\hline 40.0 & 1.5907 & 1.5907 & 1.5840 & 1.5840 & 1.5785 & 1.5785 \\
\hline 42.0 & 1.5899 & 1.5899 & 1.5831 & 1.5831 & 1.5776 & 1.5776 \\
\hline 44.1 & 1.5887 & 1.5887 & 1.5820 & 1.5820 & 1.5770 & 1.5770 \\
\hline 46.1 & 1.5876 & 1.5876 & 1.5810 & 1.5810 & 1.5758 & 1.5758 \\
\hline 48.0 & 1.5868 & 1.5868 & 1.5801 & 1.5801 & 1.5748 & 1.5748 \\
\hline 49.0 & 1.5865 & 1.5865 & 1.5797 & 1.5797 & 1.5745 & 1.5745 \\
\hline 50.0 & 1.5859 & 1.5859 & 1.5793 & 1.5793 & 1.5739 & 1.5739 \\
\hline 51.9 & 1.5850 & 1.5850 & 1.5783 & 1.5783 & 1.5729 & 1.5729 \\
\hline 54.0 & 1.5838 & 1.5838 & 1.5771 & 1.5771 & 1.5723 & 1.5723 \\
\hline
\end{tabular}
gence of UCF-35, 5CB, 5PCH, MLC-6241-000, and UCF-
TABLE I. Measured refractive indices $\left(n_{e}\right.$ and $\left.n_{0}\right)$ of $5 \mathrm{CB}$ at $\lambda=546,589$, and $633 \mathrm{~nm}$ at different temperatures.

280 measured at $\lambda=589 \mathrm{~nm}$. The filled circles, squares, open circles, filled downward triangles, and open upward triangles

\begin{tabular}{|c|c|c|c|c|c|c|}
\hline \multirow[b]{3}{*}{$T\left({ }^{\circ} \mathrm{C}\right)$} & \multicolumn{6}{|c|}{$\lambda(\mathrm{nm})$} \\
\hline & \multicolumn{2}{|c|}{546} & \multicolumn{2}{|c|}{589} & \multicolumn{2}{|c|}{633} \\
\hline & $n_{e}$ & $n_{o}$ & $n_{e}$ & $n_{o}$ & $n_{e}$ & $n_{o}$ \\
\hline 24.9 & 1.6079 & 1.4907 & 1.6036 & 1.4878 & 1.6001 & 1.4854 \\
\hline 30.1 & 1.6064 & 1.4904 & 1.6021 & 1.4873 & 1.5985 & 1.4849 \\
\hline 34.1 & 1.6032 & 1.4893 & 1.5984 & 1.4866 & 1.5944 & 1.4842 \\
\hline 39.2 & 1.5969 & 1.4890 & 1.5925 & 1.4861 & 1.5886 & 1.4835 \\
\hline 44.2 & 1.5893 & 1.4888 & 1.5848 & 1.4861 & 1.5811 & 1.4833 \\
\hline 49.3 & 1.5778 & 1.4902 & 1.5734 & 1.4875 & 1.5695 & 1.4844 \\
\hline 50.4 & 1.5738 & 1.4911 & 1.5697 & 1.4883 & 1.5665 & 1.4856 \\
\hline 51.9 & 1.5683 & 1.4933 & 1.5637 & 1.4901 & 1.5593 & 1.4869 \\
\hline 52.9 & 1.5143 & 1.5143 & 1.5109 & 1.5109 & 1.5080 & 1.5080 \\
\hline 54.2 & 1.5136 & 1.5136 & 1.5102 & 1.5102 & 1.5074 & 1.5074 \\
\hline 55.2 & 1.5131 & 1.5131 & 1.5097 & 1.5097 & 1.5068 & 1.5068 \\
\hline 56.0 & 1.5127 & 1.5127 & 1.5094 & 1.5094 & 1.5064 & 1.5064 \\
\hline 57.0 & 1.5123 & 1.5123 & 1.5089 & 1.5089 & 1.5061 & 1.5061 \\
\hline
\end{tabular}
are the measured birefringence of UCF-35, 5CB, 5PCH,

TABLE II. Measured refractive indices $\left(n_{e}\right.$ and $\left.n_{o}\right)$ of $5 \mathrm{PCH}$ at $\lambda=546,589$, and $633 \mathrm{~nm}$ at different temperatures. 
TABLE III. Measured refractive indices $\left(n_{e}\right.$ and $\left.n_{o}\right)$ of UCF-35 at $\lambda=589$, 633 , and $656 \mathrm{~nm}$ at different temperatures. Blanks in the $n_{e}$ column mean the refractive index is out of the measurement range of the Abbe refractometer.

\begin{tabular}{|c|c|c|c|c|c|c|}
\hline \multirow[b]{3}{*}{$T\left({ }^{\circ} \mathrm{C}\right)$} & \multicolumn{6}{|c|}{$\lambda(\mathrm{nm})$} \\
\hline & \multicolumn{2}{|c|}{589} & \multicolumn{2}{|c|}{633} & \multicolumn{2}{|c|}{656} \\
\hline & $n_{e}$ & $n_{o}$ & $n_{e}$ & $n_{o}$ & $n_{e}$ & $n_{o}$ \\
\hline 15 & & 1.5375 & & 1.5331 & & 1.5315 \\
\hline 20 & & 1.5372 & & 1.5329 & & 1.5306 \\
\hline 25 & & 1.5370 & & 1.5329 & & 1.5311 \\
\hline 30 & & 1.5369 & & 1.5328 & & 1.5311 \\
\hline 35 & & 1.5373 & & 1.5329 & & 1.5316 \\
\hline 40 & & 1.5377 & & 1.5331 & 1.857 & 1.5317 \\
\hline 45 & & 1.5383 & 1.8567 & 1.5336 & 1.8491 & 1.5319 \\
\hline 47.4 & 1.8674 & 1.5385 & 1.8532 & 1.5337 & 1.8450 & 1.5321 \\
\hline 50 & 1.8628 & 1.5389 & 1.8483 & 1.5343 & 1.8408 & 1.5324 \\
\hline 52.4 & 1.8585 & 1.5391 & 1.8436 & 1.5345 & 1.8364 & 1.5326 \\
\hline 55.1 & 1.8532 & 1.5398 & 1.8389 & 1.5349 & 1.8319 & 1.5329 \\
\hline 57.4 & 1.8488 & 1.5401 & 1.8348 & 1.5355 & 1.8269 & 1.5331 \\
\hline
\end{tabular}

MLC-6241-000, and UCF-280, respectively, at different temperatures. The temperature range for the experiment is from 10 to $55^{\circ} \mathrm{C}$. At room temperature and $\lambda=589 \mathrm{~nm}$, the birefringence of UCF-35, 5CB, 5PCH, MLC-6241-000, and UCF-280 is $0.35,0.2,0.12,0.086$, and 0.085 , respectively. The solid lines represent fittings using the Haller equation: ${ }^{19}$

$$
\Delta n=(\Delta n)_{o}\left(1-T / T_{c}\right)^{\beta},
$$

where $(\Delta n)_{o}$ is the LC birefringence at $T=0 \mathrm{~K}, \beta$ is a material constant, and $T_{c}$ is the clearing temperature of the LC under studies. The clearing points for UCF-35, 5CB, 5PCH, MLC-6241-000, and UCF-280 are 95.3, 33.4, 52.9, 100, and $66.2^{\circ} \mathrm{C}$, respectively. From these fittings, we find $\left[(\Delta n)_{o}, \beta\right]=[0.5727,0.2719], \quad[0.3505,0.1889], \quad[0.1706$, $0.1512],[0.1221,0.2209]$, and [0.1426, 0.2513], for UCF-35, 5CB, 5PCH, MLC-6241-000, and UCF-280, respectively. Although UCF-280 has a larger $(\Delta n)_{o}$ than MLC-6241-000,

TABLE IV. Measured refractive indices $\left(n_{e}\right.$ and $\left.n_{o}\right)$ of MLC-6241-000 at $\lambda=589,633$, and $656 \mathrm{~nm}$ at different temperatures.

\begin{tabular}{|c|c|c|c|c|c|c|}
\hline \multirow[b]{3}{*}{$T\left({ }^{\circ} \mathrm{C}\right)$} & \multicolumn{6}{|c|}{$\lambda(\mathrm{nm})$} \\
\hline & \multicolumn{2}{|c|}{589} & \multicolumn{2}{|c|}{633} & \multicolumn{2}{|c|}{656} \\
\hline & $n_{e}$ & $n_{o}$ & $n_{e}$ & $n_{o}$ & $n_{e}$ & $n_{o}$ \\
\hline 11.7 & 1.5682 & 1.4796 & 1.5657 & 1.4779 & 1.5647 & 1.4771 \\
\hline 13.5 & 1.5676 & 1.4794 & 1.5650 & 1.4772 & 1.5634 & 1.4765 \\
\hline 16.6 & 1.5661 & 1.4782 & 1.5634 & 1.4762 & 1.5619 & 1.4755 \\
\hline 21.2 & 1.5637 & 1.4768 & 1.5609 & 1.4744 & 1.5595 & 1.4738 \\
\hline 25.2 & 1.5614 & 1.4753 & 1.5587 & 1.4739 & 1.5568 & 1.4729 \\
\hline 29.9 & 1.5587 & 1.4742 & 1.5564 & 1.4724 & 1.5548 & 1.4714 \\
\hline 34.5 & 1.5557 & 1.4727 & 1.5543 & 1.4712 & 1.5509 & 1.4699 \\
\hline 39.9 & 1.5515 & 1.4706 & 1.5504 & 1.4697 & 1.5475 & 1.4683 \\
\hline 44.6 & 1.5494 & 1.4695 & 1.5472 & 1.4682 & 1.5446 & 1.4671 \\
\hline 49.4 & 1.5469 & 1.4678 & 1.5443 & 1.4662 & 1.5426 & 1.4655 \\
\hline 53.7 & 1.5443 & 1.4673 & 1.5413 & 1.4651 & 1.5395 & 1.4640 \\
\hline
\end{tabular}

TABLE V. Measured refractive indices $\left(n_{e}\right.$ and $\left.n_{o}\right)$ of UCF-280 at $\lambda=589$, 633 , and $656 \mathrm{~nm}$ at different temperatures.

\begin{tabular}{|c|c|c|c|c|c|c|}
\hline \multirow[b]{3}{*}{$T\left({ }^{\circ} \mathrm{C}\right)$} & \multicolumn{6}{|c|}{$\lambda(\mathrm{nm})$} \\
\hline & \multicolumn{2}{|c|}{589} & \multicolumn{2}{|c|}{633} & \multicolumn{2}{|c|}{656} \\
\hline & $n_{e}$ & $n_{o}$ & $n_{e}$ & $n_{o}$ & $n_{e}$ & $n_{o}$ \\
\hline 10.8 & 1.5783 & 1.4884 & 1.5751 & 1.4859 & 1.5739 & 1.4854 \\
\hline 13.0 & 1.5771 & 1.4877 & 1.5735 & 1.4853 & 1.5725 & 1.4846 \\
\hline 15.0 & 1.5756 & 1.4872 & 1.5720 & 1.4848 & 1.5709 & 1.4841 \\
\hline 20.0 & 1.5722 & 1.4857 & 1.5685 & 1.4836 & 1.5677 & 1.4824 \\
\hline 25.0 & 1.5682 & 1.4843 & 1.5649 & 1.4821 & 1.5638 & 1.4814 \\
\hline 30.0 & 1.5644 & 1.4833 & 1.5613 & 1.4809 & 1.5598 & 1.4800 \\
\hline 35.0 & 1.5600 & 1.4815 & 1.5569 & 1.4796 & 1.5558 & 1.4789 \\
\hline 40.0 & 1.5554 & 1.4804 & 1.5522 & 1.4781 & 1.5511 & 1.4773 \\
\hline 42.6 & 1.5529 & 1.4795 & 1.5497 & 1.4767 & 1.5484 & 1.4762 \\
\hline 45.0 & 1.5499 & 1.4788 & 1.5471 & 1.4763 & 1.5450 & 1.4754 \\
\hline 47.4 & 1.5477 & 1.4774 & 1.5445 & 1.4762 & 1.5430 & 1.4750 \\
\hline 50.0 & 1.5445 & 1.4785 & 1.5411 & 1.4758 & 1.5396 & 1.4748 \\
\hline 52.3 & 1.5413 & 1.4778 & 1.5381 & 1.4756 & 1.5370 & 1.4746 \\
\hline 55.0 & 1.5370 & 1.4777 & 1.5335 & 1.4754 & 1.5325 & 1.4743 \\
\hline
\end{tabular}

its clearing temperature is much lower. As a result, its birefringence at room temperature is lower than that of MLC6241-000 due to the order-parameter effect.

In Fig. 2, we plot the values of $\left(\left\langle n^{2}\right\rangle+2\right)$ and $(\sqrt{10}\langle n\rangle$ $-0.5)$ of $5 \mathrm{CB}$ and $5 \mathrm{PCH}$ in the same figure in order to validate Eq. (18) which is a close approximation of Vuks equation. The filled and open squares, circles, and upward triangles are the measured values of $\left\langle n^{2}\right\rangle+2$ and $\sqrt{10}\langle n\rangle-0.5$ at $\lambda=546,589$, and $633 \mathrm{~nm}$, respectively. From Fig. 2, we find that these data almost overlap each other. The difference is as small as $\sim 0.2 \%$ for $5 \mathrm{CB}$ and $\sim 0.1 \%$ for $5 \mathrm{PCH}$. On the other hand, the average refractive index $\langle n\rangle$ decreases linearly as the temperature increases, ${ }^{14}$

$$
\langle n\rangle=A-B T .
$$

From Eq. (20), the right side of Eq. (18) can be written as

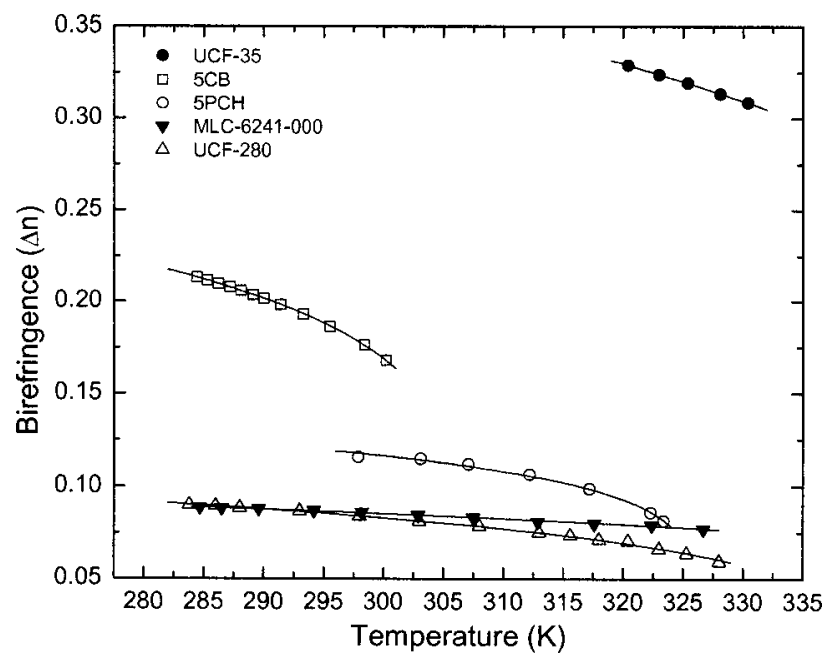

FIG. 1. Temperature-dependent birefringence $(\Delta n)$ of UCF-35 (filled circles), 5CB (open squares), 5PCH (open circles), MLC-6241-000 (filled downward triangles), and UCF-280 (open upward triangles) at $\lambda=589 \mathrm{~nm}$. The five solid lines are the fitting curves using $\Delta n=(\Delta n)_{o}\left(1-T / T_{c}\right)^{\beta}$, where $T_{c}$ is the clearing point. 


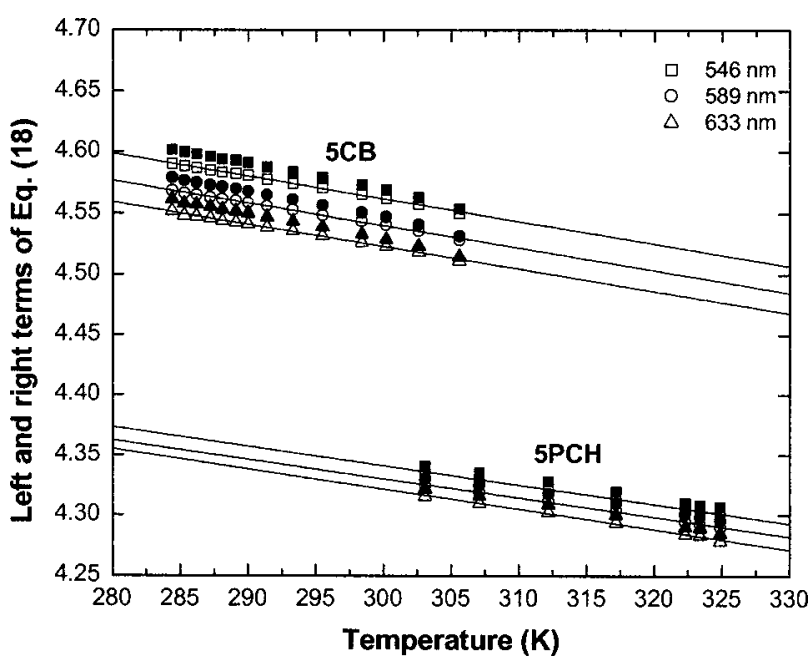

FIG. 2. Validation of Eq. (18) using the experimental data of 5CB and $5 \mathrm{PCH}$. Filled squares, circles, and upward triangles are the calculated values of $\left\langle n^{2}\right\rangle+2$ at $\lambda=546,589$, and $633 \mathrm{~nm}$, respectively. Open squares, circles, and upward triangles are the corresponding values of $\sqrt{10}\langle n\rangle-0.5$. Solid lines are fitting results using Eq. (21). The fitting parameters are [A,B] $=\left[1.7751,5.81 \times 10^{-4}\right],\left[1.7674,5.79 \times 10^{-4}\right]$, and $\left[1.7601,5.75 \times 10^{-4}\right]$ for $5 \mathrm{CB}$ at $\lambda=546,589$, and $633 \mathrm{~nm}$, respectively. The corresponding [A, B] values for $5 \mathrm{PCH}$ are $\left[1.6837,5.09 \times 10^{-4}\right],\left[1.6795,5.06 \times 10^{-4}\right]$, and $\left[1.6684,4.82 \times 10^{-4}\right]$.

$$
\sqrt{10}\langle n\rangle-0.5=\sqrt{10}(A-B T)-0.5 .
$$

The solid lines shown in Fig. 2 are the fitting results using Eq. (21). From Fig. 2, the agreement between experiment and fitting results is excellent for $5 \mathrm{CB}$ and $5 \mathrm{PCH}$. The experimental data measured at different wavelengths and temperatures of 5CB and 5PCH all satisfy Eq. (18) very well. Therefore, Vuks equation is proven to be self-consistent for describing the refractive indices of LC compounds (5CB and $5 \mathrm{PCH})$, although the isotropic local field is assumed.

Figure 3 shows a similar plot for UCF-35, MLC-6241000, and UCF-280. The filled squares, circles, and upward triangles are the measured values for $\left\langle n^{2}\right\rangle+2$ at $\lambda=656 \mathrm{~nm}$ for UCF-35, MLC-6241-000, and UCF-280, respectively. The open squares, circles, and upward triangles are the corresponding values for $\sqrt{10}\langle n\rangle-0.5$ at $\lambda=656 \mathrm{~nm}$. In Fig. 3, we plot the data at $\lambda=656 \mathrm{~nm}$ because UCF-35 has more complete experimental data at this wavelength. The refractive indices of these three LC mixtures vary from $\sim 1.46$ to $\sim 1.86$, as listed in Tables I-V. Despite such variation, Eqs. (18) and (21) still hold very well.

In Fig. 3, all the experimental data of the left and right terms of Eq. (18) overlap quite well for UCF-35, MLC-6241000, and UCF-280 although UCF-35 has $4 \times$ higher birefringence than the other two mixtures. The difference between the right- and left-hand terms of Eq. (18) is as small as $\sim 0.5 \%$ for UCF-35 and $\sim 0.1 \%$ for MLC-6241-000 and UCF-280. Solid lines are the fitting results using Eq. (21). The fitting results agree very well with the experimental data. Therefore, Vuks equation is proven to be self-consistent for both high and low birefringence LC mixtures.

As shown in Figs. 2 and 3, the $\left\langle n^{2}\right\rangle+2$ term is slightly larger than the $\sqrt{10}\langle n\rangle-0.5$ term. This is because we have omitted the higher order terms while deriving Eqs. (11a) and

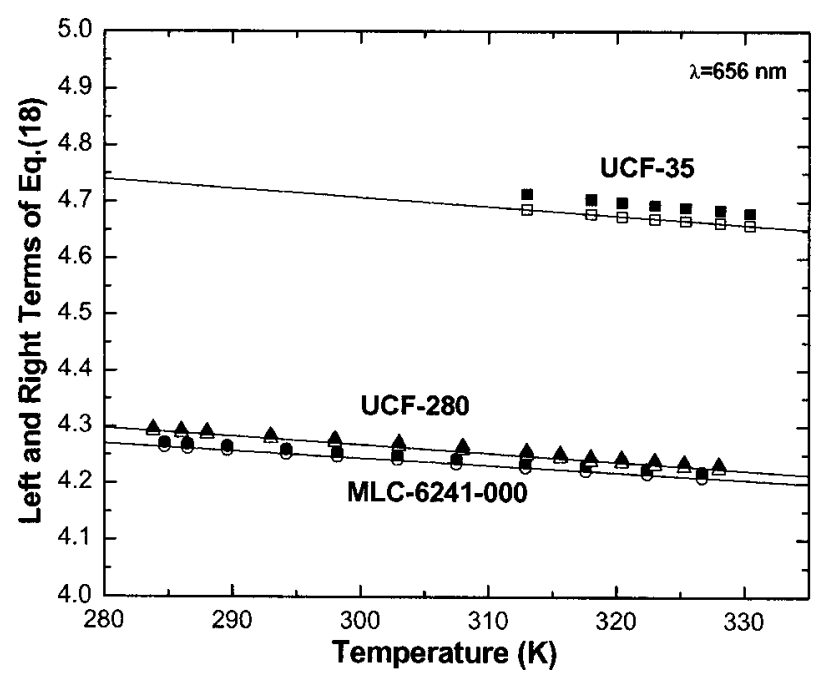

FIG. 3. Validation of Eq. (18) using the experimental data of UCF-35, UCF-280, and MLC-6241-000. Filled squares, circles, and upward triangles are the calculated values of $\left\langle n^{2}\right\rangle+2$ at $\lambda=656 \mathrm{~nm}$ for UCF-35, MLC-6241000 , and UCF-280, respectively. Open squares, circles, and upward triangles are the calculated values of the corresponding values of $\sqrt{10}\langle n\rangle-0.5$. Solid lines are fitting results using Eq. (21). The fitting parameters are [A,B] $=\left[1.8003,5.12 \times 10^{-4}\right],\left[1.6216,4.05 \times 10^{-4}\right]$, and $\left[1.6511,4.79 \times 10^{-4}\right]$ for UCF-35, MLC-6241-000, and UCF-280, respectively.

(11b) which, in turn, lead to Eqs. (16) and (18). The five LC materials we selected have refractive index spanning from $\sim 1.46$ to $\sim 1.86$. All the experimental data satisfy Eq. (18) quite well. It implies that the Vuks equation is valid for describing LC refractive indices independent of their molecular structures and electron conjugation length.

Since Vuks equation correlates the macroscopic refractive index to the microscopic molecular polarizability, if we know refractive index, then we can calculate the molecular polarizability or vice versa. For instance, if we plug the measured $n_{e}$ and $n_{o}$ data listed in Tables I-V back to Eq. (4), we can calculate the $\alpha_{e}$ and $\alpha_{o}$ values of the five LC compounds and mixtures at different temperatures and wavelengths.

In Eq. (4), there is still an unknown parameter $N$, the number of molecules per unit volume. However, $N$ is equal to $\rho N_{A} / M$, where $\rho$ is the LC density, $M$ is the molecular weight, and $N_{A}$ is the Avogadro's number. Rearranging Eq. (4), we find

$$
\begin{aligned}
& \alpha_{e}=\frac{3 M}{4 \pi \rho N_{A}} \cdot \frac{n_{e}{ }^{2}-1}{\left\langle n^{2}\right\rangle+2}, \\
& \alpha_{o}=\frac{3 M}{4 \pi \rho N_{A}} \cdot \frac{n_{o}{ }^{2}-1}{\left\langle n^{2}\right\rangle+2} .
\end{aligned}
$$

Let us use $5 \mathrm{CB}$ as an example to calculate the molecular polarizabilities, $\alpha_{e}$ and $\alpha_{o}$. For 5CB, the molecular weight is $M=249.3 \mathrm{~g} / \mathrm{mol}$ and the density $\rho(T)$ is taken from that measured by Zeller. ${ }^{20}$ Using our measured refractive indices at $\lambda=589 \mathrm{~nm}$, we can calculate the $\alpha_{e}$ and $\alpha_{o}$ of 5CB from Eq. (22).

Figure 4 plots the temperature-dependent $\alpha_{e}, \alpha_{o}$, and $\langle\alpha\rangle$ of $5 \mathrm{CB}$ at $\lambda=589 \mathrm{~nm}$. The open and filled circles represent the calculated values for $\alpha_{e}$ and $\alpha_{o}$, respectively. In the isotropic state, $\alpha_{e}$ and $\alpha_{o}$ are equal. The open triangles represent 


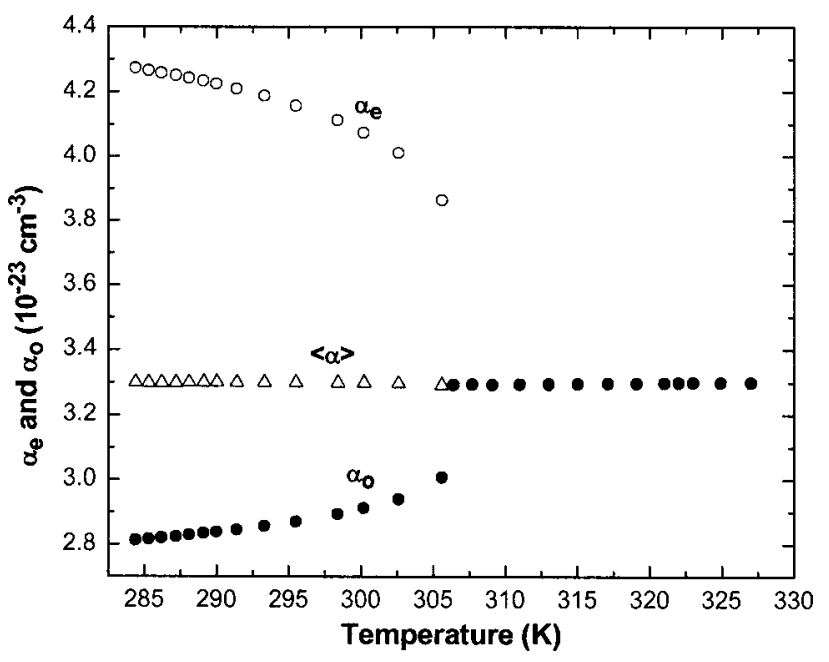

FIG. 4. Temperature-dependent molecular polarizabilites, $\alpha_{e}$ and $\alpha_{o}$, of 5CB at $\lambda=589 \mathrm{~nm}$. Open and filled circles are the results for $\alpha_{e}$ and $\alpha_{o}$, respectively. Triangles represent the average polarizability $\langle\alpha\rangle$. In the isotropic phase, $\alpha_{e}=\alpha_{o}$.

the calculated average polarizability $\langle\alpha\rangle$ in the nematic phase. From Fig. $4, \alpha_{e}$ decreases while $\alpha_{o}$ increases as the temperature increases. However, the average polarizability $\langle\alpha\rangle$ is quite insensitive to the temperature. The average polarizability for $5 \mathrm{CB}$ at $\lambda=589 \mathrm{~nm}$ is found to be $\langle\alpha\rangle \sim 3.3$ $\times 10^{-23} \mathrm{~cm}^{-3}$, which agrees very well with the calculated value $\left(\langle\alpha\rangle \sim 3.25 \times 10^{-23} \mathrm{~cm}^{-3}\right)$ published by Sarkar et al. ${ }^{21}$

\section{CONCLUSIONS}

We have developed a simple method for validating Vuks equations. By decoupling $n_{e}$ from $n_{o}$, we derived Vuks equations in a different form. We are able to correlate the molecular polarizabilities $\alpha_{e, o}$ to another macroscopic term $\langle n\rangle$. Through the derivation process, we find another relationship between $\left\langle n^{2}\right\rangle$ and $\langle n\rangle$, as described by Eq. (18). Using our experimental results, Vuks equation is validated despite that the isotropic local field is assumed. Based on Vuks equations, the molecular polarizabilities and average polarizability of $5 \mathrm{CB}$ at $\lambda=589 \mathrm{~nm}$ are obtained in the nematic and isotropic phases.

\section{ACKNOWLEDGMENTS}

The authors are indebted to Dr. S. Gauza for his technical support. This work is supported by AFOSR under Contract No. F49620-01-1-0377.

${ }^{1}$ S. T. Wu and D. K. Yang, Reflective Liquid Crystal Displays (Wiley, New York, 2001).

${ }^{2}$ P. F. McManamon et al., Proc. IEEE 84, 268 (1996).

${ }^{3}$ H. Ren, Y. H. Fan, and S. T. Wu, Appl. Phys. Lett. 82, 3168 (2003).

${ }^{4}$ N. V. Tabiryan, A. V. Sukhov, and B. Ya. Zel'dovich, Mol. Cryst. Liq. Cryst. 136, 1 (1986)

${ }^{5}$ S. T. Wu, C. S. Wu, M. Warenghem, and M. Ismaili, Opt. Eng. 32, 1775 (1993).

${ }^{6}$ M. F. Vuks, Opt. Spektrosk. 20, 644 (1966).

${ }^{7}$ G. Vertogen and W. H. de Jeu, Thermotropic Liquid Crystals (Springer Verlag, Berlin, 1988), Chap. 10

${ }^{8}$ S. T. Wu, Phys. Rev. A 33, 1270 (1986).

${ }^{9}$ S. T. Wu, J. Appl. Phys. 69, 2080 (1991).

${ }^{10}$ I. Abdulhalim, Mol. Cryst. Liq. Cryst. 197, 103 (1991).

${ }^{11}$ I. C. Khoo and S. T. Wu, Optics and Nonlinear Optics of Liquid Crystals (World Scientific, Singapore, 1993).

${ }^{12}$ E. M. Averyanov, J. Opt. Technol. 64, 417 (1997).

${ }^{13}$ J. Li and S. T. Wu, J. Appl. Phys. 95, 896 (2004).

${ }^{14} \mathrm{~J}$. Li, S. Gauza, and S. T. Wu, J. Appl. Phys. 96, 19 (2004).

${ }^{15}$ For example, see J. D. Jackson, Classical Electrodynamics, 2nd ed. (Wiley, New York, 1962).

${ }^{16}$ C. Bunn and R. Daubeny, Trans. Faraday Soc. 50, 1173 (1954).

${ }^{17}$ A. D. Buckingham, in Molecular Electro-optics, edited by C. T. O'Konski (Marcel Dekker, New York, 1976), Chap. 2.

${ }^{18}$ L. M. Blinov, Electro-optical and Magneto-optical Properties of Liquid Crystals (Wiley, New York, 1983), Chap. 2.

${ }^{19}$ I. Haller, Prog. Solid State Chem. 10, 103 (1975).

${ }^{20}$ H. R. Zeller, Phys. Rev. A 26, 1785 (1982).

${ }^{21}$ P. Sarkar, P. Mandal, S. Paul, and R. Paul, Liq. Cryst. 30, 507 (2003). 